\title{
Comparison between the Effects of Loxoprofen and Acetaminophen on Postoperative Pain Following Radical Prostatectomy: A Propensity Score Matching Analysis
}

\author{
Fumihiro Nishimura, ${ }^{*, a}$ Tomoko Ushijima, ${ }^{a}$ Masako Nojima,${ }^{b}$ Shinsuke Hamada,${ }^{c}$ \\ Kazumasa Hara, ${ }^{c}$ Yasuyuki Hamada ${ }^{c}$ Daisuke Kadowaki, ${ }^{d}$ Shigeyuki Miyamura, ${ }^{d}$ \\ Kentaro Oniki, ${ }^{e}$ and Junji Saruwatari ${ }^{e}$ \\ ${ }^{a}$ Department of Clinical Pharmacy, Kumamoto Chuo Hospital; 1-5-1 Tainoshima, Minami-ku, Kumamoto 862-0965, \\ Japan: ${ }^{b}$ Department of Nursing, Kumamoto Chuo Hospital; 1-5-1 Tainoshima, Minami-ku, Kumamoto 862-0965, \\ Japan: ${ }^{c}$ Department of Urology Kumamoto Chuo Hospital; 1-5-1 Tainoshima, Minami-ku, Kumamoto 862-0965, \\ Japan: ${ }^{d}$ Faculty of Pharmaceutical Sciences, Sojo University; 4-22-1 Ikeda, Nishi-ku, Kumamoto 860-0082, \\ Japan: and ${ }^{e}$ Division of Pharmacology and Therapeutics, Graduate School of Pharmaceutical Sciences, \\ Kumamoto University; 5-1 Oe-honmachi, Chuo-ku, Kumamoto 862-0973, Japan. \\ Received March 9, 2021; accepted July 2, 2021
}

\begin{abstract}
Non-steroidal anti-inflammatory medications are associated with renal impairment. However, there is little information on whether these medications affect postoperative renal function compared with acetaminophen. The objective of this study was to compare the effects of acetaminophen and loxoprofen, used as postoperative analgesic, effect on postoperative analgesia using propensity score matching analysis. We retrospectively enrolled 328 patients treated with loxoprofen or acetaminophen after open radical prostatectomy between October 2017 and February 2020. We analyzed postoperative pain intensity, the incidence rate of acute kidney injury, drug-induced liver injury, and rate of elevation in serum creatinine after open radical prostatectomy. Eighty-one matched pairs of patients treated with loxoprofen or acetaminophen were selected using propensity score matching analysis. The postoperative numerical rating scale was significantly higher in the acetaminophen group than in the loxoprofen group on postoperative day 5 . The use of patientcontrolled anesthesia and rescue analgesics was significantly higher in the acetaminophen group than in the loxoprofen group. The loxoprofen group had a significantly higher postoperative increase in serum creatinine than the acetaminophen group on postoperative days 5 and 8 . The incidence of acute kidney injury was $4.9 \%$ in the loxoprofen group and $0 \%$ in the acetaminophen group, while the incidence of drug-induced liver injury was $0 \%$ in both groups. Acetaminophen appears to be safer than loxoprofen in terms of effects on renal function. Nevertheless, the number of acetaminophen doses and the dose per dose may need to be increased for patients with significant postoperative pain.
\end{abstract}

Key words radical prostatectomy; postoperative pain; non-steroidal anti-inflammatory drug; loxoprofen; acetaminophen

\section{INTRODUCTION}

Radical prostatectomy is one of the most widely used treatment options for localized prostate cancer and is generally performed as either robot-assisted radical prostatectomy or open radical prostatectomy (ORP). ${ }^{1)}$ Non-steroidal anti-inflammatory drugs (NSAIDs) are used to treat mild-to-moderate pain as first-line postoperative analgesics and antipyretics despite their several adverse effects, including gastrointestinal disorders and renal impairment. ${ }^{2,3}$ Acute kidney injury (AKI) is a severe postoperative complication associated with increased duration of hospital stay, rates of readmission, and hospital mortality. ${ }^{4-6)}$ Minor changes in serum creatinine (SCr) levels are significantly linked to the length of stay and mortality. ${ }^{7)}$ NSAIDs reduce renal blood flow and glomerular filtration rate, leading to AKI. ${ }^{8}$ Acetaminophen ( $N$-acetylpara-amino-phenol (APAP)) is a commonly used postoperative analgesic and antipyretic. Compared to NSAIDs, APAP causes fewer adverse effects with respect to AKI; however, several investigations have shown that APAP significantly increases the risk of renal impairment. ${ }^{9)}$ Furthermore, it is unclear whether NSAIDs worsen postoperative renal function more than APAP. Therefore, it is essential to determine whether there is any relationship between postoperative analgesics and changes in SCr to achieve better postoperative pain management with fewer side-effects. In this observational study, we aimed to compare the effects of loxoprofen (LOX), an NSAID, to those of APAP on postoperative analgesic effect and postoperative renal function in patients with ORP with normal renal function using propensity score matching analysis.

\section{PATIENTS AND METHODS}

Patients' Characteristics A total of 328 patients with a normal renal function treated with LOX or APAP as postoperative analgesics after ORP between October 2017 and February 2020 at Kumamoto Chuo Hospital were enrolled in this study. The exclusion criteria were as follows: a history of preoperative NSAIDs (expect for low dose aspirin) or APAP use, administration of intravenous patient-controlled analgesia or epidural anesthesia using narcotics, transient AKI on post- 


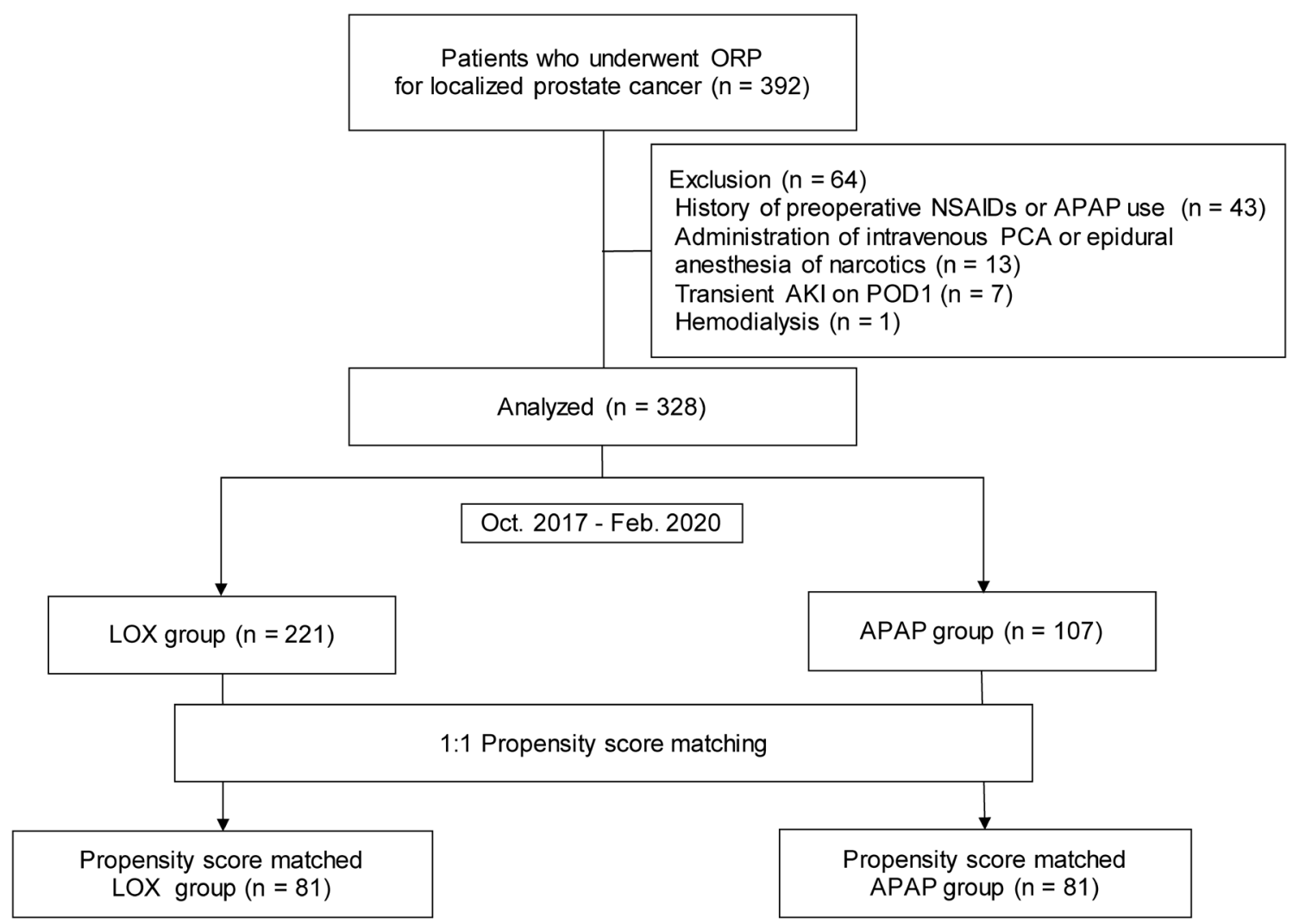

Fig. 1. Study Flow Chart

ORP open radical prostatectomy, NSAIDs non-steroidal anti-inflammatory drugs, APAP, acetaminophen; PCA, patient-controlled anesthesia; AKI, acute kidney injury; POD, postoperative day; LOX, loxoprofen.

operative day (POD) 1, hemodialysis, and incomplete information from medical records.

Clinical Data Collection Clinical data, preoperative and intraoperative variables, and postoperative laboratory values until POD 8 were collected. Preoperative variables included age, height, weight, body surface area, prostate-specific antigen, Gleason score, diabetes mellitus, hypertension, angiotensin 2 receptor blocker, an angiotensin-converting enzyme inhibitor, low dose aspirin, anticoagulant, serum albumin, hemoglobin, platelets, estimated glomerular filtration rate, $\mathrm{SCr}$, total bilirubin (T-bil), alkaline phosphatase (ALP), and alanine aminotransferase (ALT). Intraoperative variables included operation time, anesthesia time, blood loss, autologous blood transfusion, red blood cell transfusion, epidural anesthesia, and hydroxyethyl starch dosage per body weight. Postoperative blood tests were drawn between 8 and $10 \mathrm{a} . \mathrm{m}$. on PODs 1,5 , and 8 . The intensity of postoperative pain was evaluated by a nurse on PODs 1, 3, 5, and 7 on the Numerical Rating Scale (NRS). The NRS was reported to be well matched in assessing acute postoperative pain, comparable in sensitivity to well-known visual analogue scale for assessing pain intensity, and superior to the four-point verbal categorical rating scale. ${ }^{10)}$

\section{General Postoperative Pain Management}

Epidural Anesthesia

Epidural anesthesia was administered as a continuous infusion of $300 \mathrm{~mL}$ of $0.2 \%$ ropivacaine or $0.17 \%$ levobupivacaine at a rate of $3-5 \mathrm{~mL} / \mathrm{h}$ intraoperatively along with $3 \mathrm{~mL}$ patientcontrolled anesthesia (PCA) (Smiths Medical, Tokyo, Japan) as rescue analgesia until the POD 3.
Treatment with LOX and APAP

On POD 1, the LOX and APAP groups were administered one $60 \mathrm{mg}$ tablet/dose thrice daily and one $500 \mathrm{mg}$ tablet/dose thrice daily (after each meal), respectively. They were administered LOX $60 \mathrm{mg}$ (LOX group only) and APAP $500 \mathrm{mg}$ (APAP group only) orally, or pentazocine $15 \mathrm{mg}$ or APAP 500-1000 mg (APAP group only) intravenously as rescue medications. Postoperative analgesics were continued for at least one week until postoperative pain was sufficiently controlled.

Primary and Secondary Endpoints

The primary endpoint of this study was postoperative pain intensity. Postoperative pain intensity was recorded using NRS at PODs 1, 3, 5, and 7 after ORP while the patient was at rest. When the patient required a rescue analgesic for postoperative pain, the rescue medication was administered. The secondary endpoints were the rate of increase in $\mathrm{SCr}$ in the LOX and APAP groups within $8 \mathrm{~d}$ of ORP, the incidence of AKI and drug-induced liver injury (DILI) within $7 \mathrm{~d}$ of postoperative analgesics, the number of PCA pushes, the percentage of patients using postoperative rescue analgesics, and the length of hospital stay. The rate of increase in SCr was defined as postoperative $\mathrm{SCr}$ after $\mathrm{ORP}) /($ preoperative baseline SCr. According to the Kidney Disease: Improving Global Outcomes criteria, AKI was defined as SCr elevation of $\geq 1.5$ times within the previous $7 \mathrm{~d}^{11}{ }^{11}$ The Kidney Disease: Improving Global Outcomes criteria define stage $1 \mathrm{AKI}$ as $\mathrm{SCr}$ elevation of $\geq 0.3 \mathrm{mg} / \mathrm{dL}$ within $48 \mathrm{~h}$ or $\geq 1.5$ times within the previous $7 \mathrm{~d}$; in our study, $\mathrm{SCr}$ was measured as preoperative baseline $\mathrm{SCr}$, and on PODs 1, 5, and 8. Accordingly, we used only $\mathrm{SCr}$ elevation as the rate of increase in SCr. DILI is a 
Table 1. Preoperative and Intraoperative Characteristics of Patients with Open Radical Prostatectomy before and after the Propensity Score Matching

\begin{tabular}{|c|c|c|c|c|c|c|c|}
\hline & \multicolumn{2}{|c|}{ All patients } & \multirow{2}{*}{$p$-Value } & \multicolumn{2}{|c|}{ Propensity score matching patients } & \multirow{2}{*}{$p$-Value } & \multirow{2}{*}{ Std diff } \\
\hline & $\operatorname{LOX}(n=221)$ & $\operatorname{APAP}(n=107)$ & & $\operatorname{LOX}(n=81)$ & $\operatorname{APAP}(n=81)$ & & \\
\hline Age, years & $69 \pm 6$ & $71 \pm 5$ & 0.001 & $71 \pm 5$ & $70 \pm 6$ & 0.967 & 0.007 \\
\hline Height, m & $1.65 \pm 0.06$ & $1.65 \pm 0.06$ & 0.635 & $1.65 \pm 0.07$ & $1.65 \pm 0.06$ & 0.725 & 0.063 \\
\hline Weight, kg & $65.6 \pm 9.5$ & $65.5 \pm 10.1$ & 0.886 & $65.6 \pm 9.7$ & $65.1 \pm 10.0$ & 0.838 & 0.054 \\
\hline BSA, $\mathrm{m}^{2}$ & $1.71 \pm 0.14$ & $1.71 \pm 0.14$ & 0.979 & $1.71 \pm 0.14$ & $1.71 \pm 0.14$ & 0.941 & 0.001 \\
\hline PSA, ng/mL & $9.4 \pm 30.0$ & $8.4 \pm 12.5$ & 0.830 & $6.6 \pm 5.5$ & $6.9 \pm 6.1$ & 0.860 & 0.068 \\
\hline Gleason score & $7.4 \pm 0.8$ & $7.4 \pm 1.0$ & 0.735 & $7.4 \pm 0.8$ & $7.4 \pm 0.9$ & 0.990 & 0.057 \\
\hline Diabetes mellitus, $n(\%)$ & $42(19.0)$ & $19(17.8)$ & 0.880 & $9(11.1)$ & $11(13.6)$ & 0.812 & 0.075 \\
\hline Hypertension, $n(\%)$ & $119(53.8)$ & $67(62.6)$ & 0.154 & $45(55.6)$ & $46(56.8)$ & 1.000 & 0.025 \\
\hline $\mathrm{ARB}, n(\%)$ & $77(34.8)$ & $36(33.6)$ & 0.902 & $25(30.9)$ & $24(29.6)$ & 1.000 & 0.027 \\
\hline $\mathrm{ACE}, n(\%)$ & $9(4.1)$ & $6(5.6)$ & 0.677 & $4(4.9)$ & $3(3.7)$ & 1.000 & 0.027 \\
\hline Low dose aspirin, $n(\%)$ & $22(10.0)$ & $3(2.8)$ & 0.025 & $4(4.9)$ & $3(3.7)$ & 1.000 & 0.061 \\
\hline Anticoagulant, $n(\%)$ & $3(1.4)$ & $6(5.6)$ & 0.063 & $2(2.5)$ & $4(4.9)$ & 0.682 & 0.131 \\
\hline \multicolumn{8}{|l|}{ Preoperative laboratory variables } \\
\hline Serum albumin, g/dL & $4.3 \pm 0.3$ & $4.2 \pm 0.3$ & 0.016 & $4.2 \pm 0.3$ & $4.2 \pm 0.3$ & 0.958 & 0.050 \\
\hline Hemoglobin, g/dL & $13.7 \pm 1.1$ & $13.8 \pm 1.4$ & 0.358 & $14.0 \pm 1.1$ & $13.7 \pm 1.3$ & 0.060 & 0.251 \\
\hline Platelet, $\times 10^{3} / \mu \mathrm{L}$ & $22.1 \pm 12.2$ & $21.1 \pm 5.0$ & 0.669 & $21.2 \pm 5.6$ & $21.7 \pm 5.1$ & 0.417 & 0.104 \\
\hline $\mathrm{e}-\mathrm{GFR}, \mathrm{mL} / \mathrm{min} / 1.73 \mathrm{~m}^{2}$ & $71.0 \pm 12.2$ & $64.3 \pm 16.6$ & $<0.001$ & $68.2 \pm 14.2$ & $69.3 \pm 14.3$ & 0.458 & 0.079 \\
\hline $\mathrm{SCr}, \mathrm{mg} / \mathrm{dL}$ & $0.84 \pm 0.13$ & $0.85 \pm 0.23$ & $<0.001$ & $0.88 \pm 0.15$ & $0.87 \pm 0.16$ & 0.485 & 0.068 \\
\hline T-bil, mg/dL & $0.80 \pm 0.30$ & $0.77 \pm 0.33$ & 0.118 & $0.80 \pm 0.36$ & $0.79 \pm 0.35$ & 0.555 & 0.045 \\
\hline $\mathrm{ALP}, \mathrm{U} / \mathrm{L}$ & $215.8 \pm 58.1$ & $224.9 \pm 65.6$ & 0.308 & $222.4 \pm 61.9$ & $228.3 \pm 71.0$ & 0.738 & 0.088 \\
\hline $\mathrm{ALT}, \mathrm{U} / \mathrm{L}$ & $22.4 \pm 10.9$ & $24.3 \pm 14.5$ & 0.172 & $24.7 \pm 14.1$ & $25.4 \pm 15.9$ & 0.605 & 0.047 \\
\hline \multicolumn{8}{|l|}{ Intraoperative variables } \\
\hline Operation time, $\min$ & $96 \pm 28$ & $97 \pm 27$ & 0.918 & $97 \pm 28$ & $94 \pm 29$ & 0.590 & 0.100 \\
\hline Anesthesia time, min & $137 \pm 31$ & $139 \pm 30$ & 0.589 & $135 \pm 32$ & $138 \pm 31$ & 0.582 & 0.087 \\
\hline blood loss, $\mathrm{mL}$ & $377 \pm 255$ & $324 \pm 209$ & 0.047 & $359 \pm 240$ & $327 \pm 215$ & 0.407 & 0.139 \\
\hline Autologous blood transfusion, $n(\%)$ & $219(99.1)$ & $106(99.1)$ & 1.000 & $81(100)$ & $80(98.8)$ & 1.000 & 0.158 \\
\hline Red blood cell transfusion, $n(\%)$ & $2(0.9)$ & $1(0.9)$ & 1.000 & $0(0)$ & $1(1.2)$ & 1.000 & 0.159 \\
\hline Hydroxyethyl starch, mL/kg & $1.79 \pm 3.51$ & $1.16 \pm 2.52$ & 0.163 & $0.82 \pm 2.15$ & $1.22 \pm 2.56$ & 0.278 & 0.169 \\
\hline \multicolumn{8}{|l|}{ Epidural anesthesia } \\
\hline $0.2 \%$ Ropivacaine $(3 \mathrm{~mL} / \mathrm{h}), n(\%)$ & $7(3.2)$ & $3(2.8)$ & 1.000 & $3(3.7)$ & $2(2.5)$ & 1.000 & 0.071 \\
\hline $0.2 \%$ Ropivacaine $(4 \mathrm{~mL} / \mathrm{h}), n(\%)$ & $20(9.0)$ & $10(9.3)$ & 1.000 & $11(13.6)$ & $8(9.9)$ & 0.626 & 0.115 \\
\hline $0.2 \%$ Ropivacaine $(5 \mathrm{~mL} / \mathrm{h}), n(\%)$ & $158(71.5)$ & $74(69.2)$ & 0.699 & $56(69.1)$ & $58(71.6)$ & 0.864 & 0.054 \\
\hline $0.17 \%$ Levobupivacaine $(5 \mathrm{~mL} / \mathrm{h}), n(\%)$ & $36(16.3)$ & $20(18.7)$ & 0.639 & $11(13.6)$ & $13(16.0)$ & 0.825 & 0.070 \\
\hline
\end{tabular}

Data were expressed as mean \pm standard deviation and number (percentage) of patients as appropriate. LOX, loxoprofen; APAP, acetaminophen; Std diff, standardized differences; BSA, body surface area; PSA, prostate-specific antigen; ARB, angiotensin 2 receptor blocker; ACE, angiotensin-converting enzyme inhibitor; e-GFR, estimated glomerular filtration rate; SCr, serum creatinine; T-bil, total bilirubin; ALP, alkaline phosphatase; ALT, alanine aminotransferase.

clinical criterion adopted in 2011 and is defined as any one of the following: an ALT level $\geq 5 \times$ the upper limit of normal (ULN) of $42 \mathrm{IU} / \mathrm{L}$; an ALP level $\geq 2 \times \mathrm{ULN}$ of $322 \mathrm{IU} / \mathrm{L}$; or an ALT level $\geq 3 \times$ ULN of 42 IU/L plus a T-bil level $\geq 2 \times U L N$ of $1.5 \mathrm{mg} / \mathrm{dL}{ }^{12)}$

Ethics Approval This study protocol followed the ethical guidelines of the 1975 Declaration of Helsinki and the current ethical guidelines. The study was approved by the Institutional Ethics Committee of Kumamoto Chuo Hospital (Approval No. 73-01).

Data Analysis Patient characteristics were expressed as mean \pm standard deviation and number (percentage) of patients as appropriate. Data variables were compared between the LOX and APAP groups using the Mann-Whitney $U$ test for continuous variables and the Fischer test for categorical variables. A multivariable logistic regression analysis was performed to determine the propensity score using the following 24 variables at baseline: age; height; weight; body surface area; prostate-specific antigen; Gleason score; the prevalence of diabetes mellitus and hypertension; use of angiotensin 2 receptor blocker, an angiotensin-converting enzyme inhibitor, low dose aspirin, and anticoagulant; preoperative laboratory values (serum albumin, hemoglobin, platelets, estimated glomerular filtration rate, and $\mathrm{SCr}$ ); and intraoperative variables including operation time, anesthesia time, blood loss, autologous blood transfusion, red blood cell transfusion, epidural anesthesia, and hydroxyethyl starch dosage per body weight. The $1: 1$ propensity score matching was performed using nearest neighbor matching with a maximum caliper width of 0.2 standard deviations of the propensity scores. This model's conformance was examined using the Hosmer-Lemeshow goodness of fit, C-statistics index, and standardized difference. Results with $p$-value $<0.05$ were considered significant. All statistical analyses were performed using SPSS for Windows (v25; IBM Japan, Tokyo, Japan).

\section{RESULTS}

Patients' Characteristics During this study period, all patients who started treatment with LOX $(n=221)$ and APAP $(n=107)$ were enrolled after scrutiny based on the exclusion criteria (Fig. 1). After matching the propensity score, 81 
matched pairs of patients were determined. Characteristics at baseline before and after propensity score matching are shown in Table 1. Before propensity score matching analysis, age, low dose aspirin, serum albumin, preoperative estimated glomerular filtration rate, $\mathrm{SCr}$, and intraoperative blood loss were significantly different between the LOX and APAP groups.

Propensity Score Allocation The propensity score was calculated using the preoperative and intraoperative patient characteristics. This model did not display evidence of lack of fit based on the Hosmer-Lemeshow statistic $(p=0.61)$ and validated high discriminative ability $(c=0.77)$. After propensity score matching analysis, the standardized difference was within $\pm 1.96 \times \sqrt{2 / n}$; that is 0.31 , and there was no difference between the two groups for these patient characteristics. $^{13)}$

Clinical Efficacy and Outcome The worst pain intensity during rest recorded on the NRS after ORP was shown in Fig. 2. Patients in the LOX group had a median NRS of 1.0 (0-2) pain intensity on POD 5, whereas patients in the APAP group had a significantly higher median NRS of 2.0 (1-3) pain

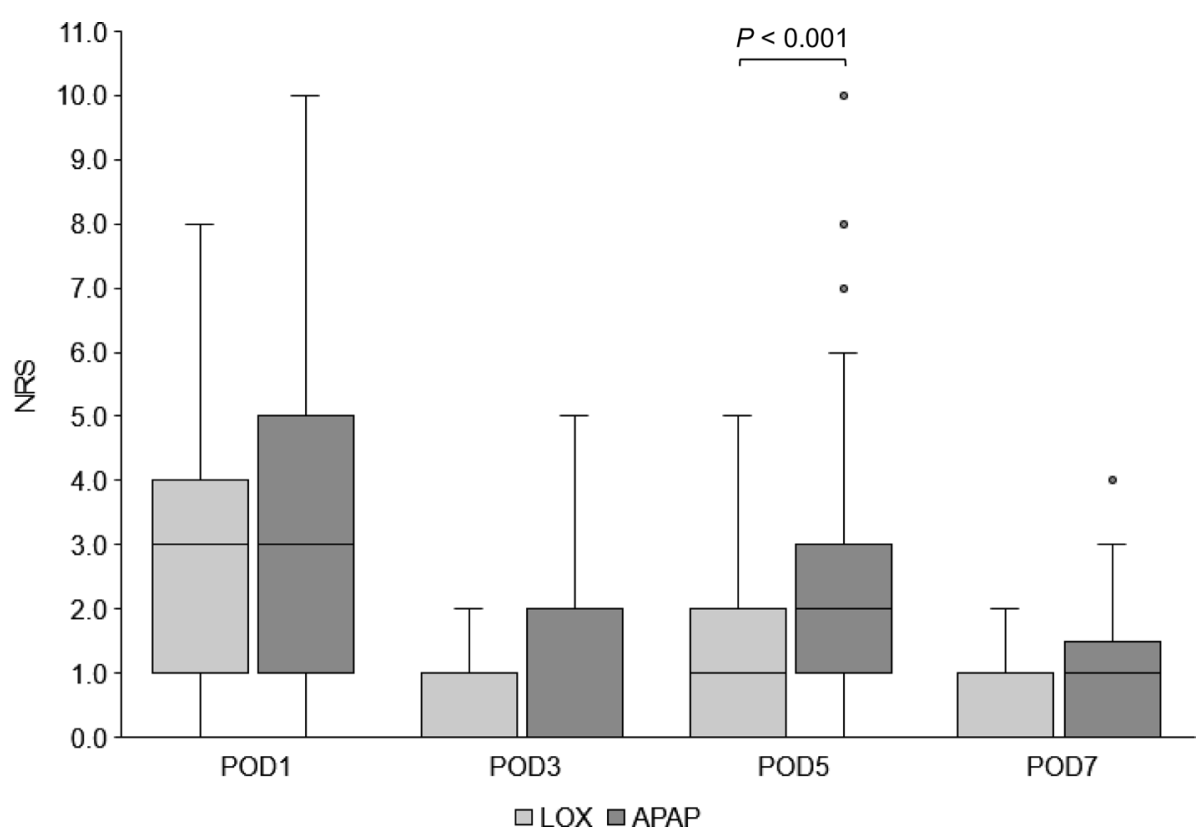

Fig. 2. Box Plots of Postoperative NRS Scores of Pain Intensity between the LOX and APAP Groups

Box plots show median, interquartile range (25-75\%), whiskers, and minimal scores. NRS, numerical rating scale; POD, postoperative day; LOX, loxoprofen; APAP, acetaminophen.

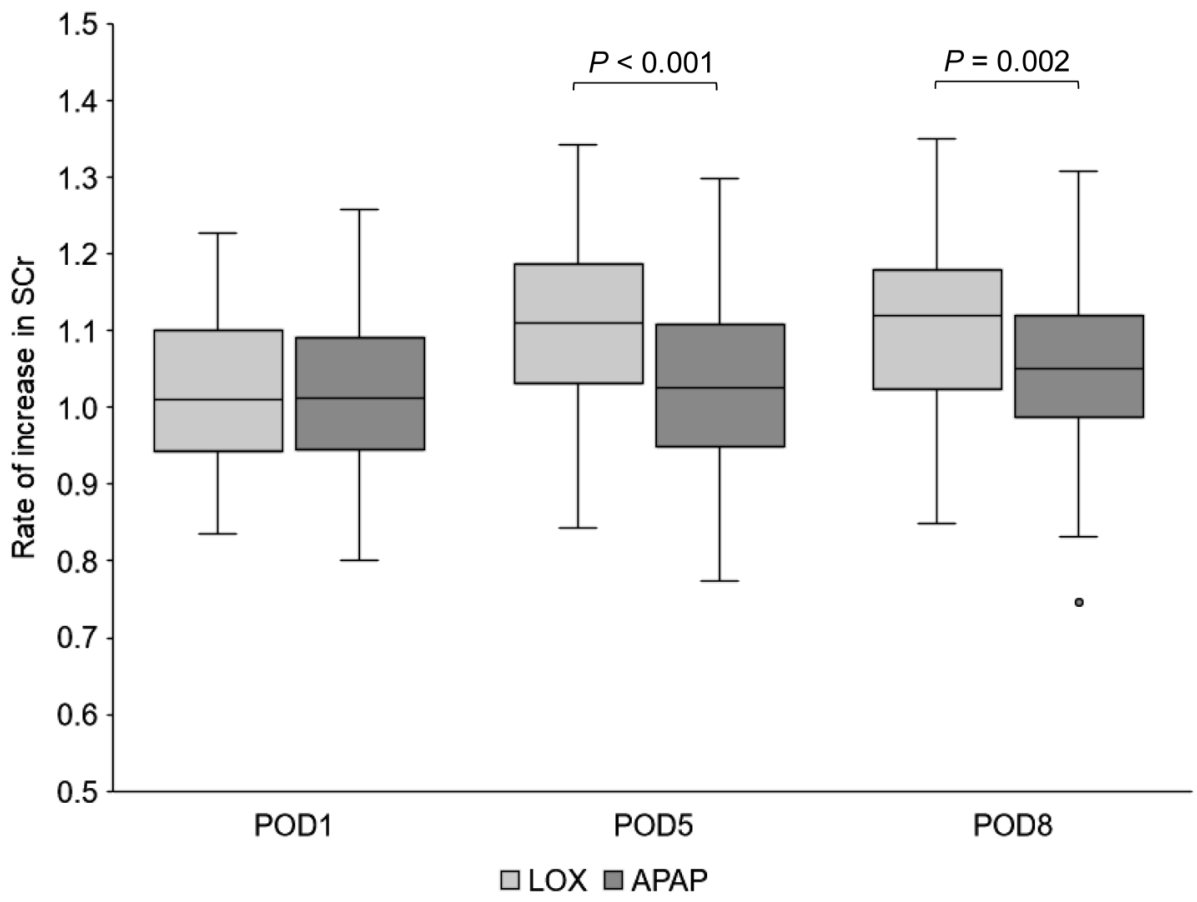

Fig. 3. Box Plots of Postoperative Rate of Increase in SCr between the LOX and APAP Groups

Box plots show median, interquartile range (25-75\%), whiskers, and minimal scores. SCr, serum creatinine; POD, postoperative day; LOX, loxoprofen; APAP, acetaminophen. 
Table 2. Comparison of the Incidences of Postoperative AKI, Number of PCA Pushes, Use of Rescue Analgesics, and Length of Hospital Stay between the LOX and APAP Groups

\begin{tabular}{lccc}
\hline \hline & $\begin{array}{c}\text { LOX } \\
(n=81)\end{array}$ & $\begin{array}{c}\text { ASAP } \\
(n=81)\end{array}$ & $p$-Value \\
\hline AKI, $n(\%)$ & $2(4.9)$ & $0(0)$ & - \\
DILI, $n(\%)$ & $0(0)$ & $0(0)$ & - \\
Number of PCA pushes, times & $1.2 \pm 1.4$ & $3.3 \pm 3.5$ & $<0.001$ \\
Use of rescue analgesics, $n(\%)$ & $23(28.4)$ & $41(50.6)$ & $<0.001$ \\
Length of hospital stay, days & $15.1 \pm 4.1$ & $18.8 \pm 7.6$ & 0.836 \\
\hline
\end{tabular}

Data were expressed as mean \pm standard deviation and number (percentage) of patients as appropriate. LOX, loxoprofen; APAP, acetaminophen; AKI, acute kidney injury; DILI, drug-induced liver injury; PCA, patient-controlled anesthesia.

intensity on POD $5(p<0.001)$ (Fig. 2). The postoperative increase rate in $\mathrm{SCr}$ after ORP was shown in Fig. 3. Patients in the APAP group had a median increase rate in $\mathrm{SCr}$ of 1.01 (0.94-1.10) and $1.05(0.98-1.12)$ for POD5 and POD8, respectively, whereas patients in the LOX group had a significantly higher median increase rate in SCr of $1.11(1.05-1.19)$ and 1.14 (1.06-1.20) for POD5 and POD8, respectively $(p<0.001$, $p=0.002$ ) (Fig. 3). The incidence of AKI was four cases $(4.9 \%)$ in the LOX group and none in the APAP group, and no DILI occurred in either group (Table 2). The number of PCA pushes and the percentage of patients using postoperative rescue analgesics was significantly higher in the APAP group than in the LOX group; however, there was no difference in the length of hospital stay (Table 2). The only drug that affected liver or kidney function that was initiated after POD1 was injectable cephem antibiotics (8 patients in the LOX group, 13 patients in the APAP group, $p=0.35$ ).

\section{DISCUSSION}

We performed this study to examine the possible association between postoperative LOX administration and postoperative pain compared with the APAP group. There were significant differences between postoperative analgesic effects in the LOX group and the APAP group on POD 5 only. A significant difference was observed only on postoperative day 5, suggesting that epidural anesthesia was administered until postoperative day 3 and that acetaminophen alone was less effective in the early postoperative period. A previous pharmacokinetic simulation of APAP in Japanese patients with chronic pain reported that $500 \mathrm{mg} 3$ times a day of APAP relieved pain for only $10.2 \mathrm{~h}^{14)}$ In addition, previous randomized trials indicated that LOX was slightly stronger than APAP in analgesic effect after arthroscopic knee surgery. ${ }^{15)}$ Clinical practice guidelines for pain management indicate that patients with significant pain (NRS $\geq 4$ ) should receive analgesia therapy. ${ }^{16)}$ As our findings indicated that the use of PCA and the percentage of patients using postoperative rescue analgesics in the APAP group were higher than those in the LOX group, indicating use use more frequent administration of APAP and a higher single dose of APAP is necessary to maintain the analgesic effect.

In contrast, our results showed that the increase in $\mathrm{SCr}$ after ORP was significantly higher in the LOX group than in the APAP group. Moreover, the incidence of AKI tended to be higher in the LOX group than in the APAP group. In this study, $p$ value could not be calculated because the occurrence of AKI in the APAP group was $0 \%$; this may be due to lack of analytical power, given the small number of patients with AKI. Previous studies have reported that risk factors for AKI patient characteristics include aging, hypertension, diabetes, anemia, heart failure, and preoperative renal function. ${ }^{17)}$ There were no patients with heart failure in this study. In addition, medications at risk for AKI include reninangiotensin system inhibitors, aminoglycosides, diuretics and anticoagulants such as warfarin and direct oral anticoagulants. ${ }^{18,19)}$ In this study, no patient received aminoglycosides or diuretics before or after ORP. The only drug used after POD1 that was thought to affect renal or hepatic function was injectable cephem antibiotics, and there was no significant difference between the number of patients who received these antibiotics in the two groups. Preoperative prostate-specific antigen and Gleason scores have also been reported to affect postoperative complications ${ }^{20)}$; propensity score matching may have minimized the impact of postoperative complications. Recent investigations reported that intraoperative bleeding is a significant risk factor for AKI following ORP. ${ }^{21)}$ In this study, intraoperative bleeding was not significantly different after propensity score matching analysis. A previous metaanalysis showed that fluid containing high chloride content in perioperative fluid management was associated with increased $\mathrm{AKI}^{22}{ }^{22}$ In this study, oral intake in the postoperative period was started on POD 1 in almost all cases. Moreover, $1.5 \mathrm{~L}$ Ringer's acetate (130.0 Na and $109.0 \mathrm{Cl}$, in $\mathrm{mmol} / \mathrm{L})$ and $1 \mathrm{~L}$ hypotonic maintenance fluid $(35.0 \mathrm{Na}$ and $35.0 \mathrm{Cl}$, in $\mathrm{mmol} / \mathrm{L}$ ) with $4.3 \%$ glucose were administered from POD 0 to 1 in all cases. A systematic review showed that postoperative NSAID administration may be associated with increases in $\mathrm{SCr}^{23}$ ) A recent prospective cohort study reported that very small increases in SCr were involved in mortality. ${ }^{24)}$ Therefore, preventing an increase in SCr is very important. The risk of renal impairment caused by APAP has been controversial., ${ }^{9,25}$ Our findings indicate that the postoperative increase in the rate of $\mathrm{SCr}$ in patients administered LOX was higher than that in patients administered APAP; therefore, APAP could be used safely in patients undergoing surgery.

There were several limitations to this study. First, the propensity score matching analysis could reduce selection bias by creating a pseudo-random assignment-like situation in observational studies. However, the propensity score matching analysis results were universalizable only within the range of propensity scores, and they could not be applied outside the propensity score range. A potential limitation is that the unknown confounders could not be removed by propensity score matching analysis. The results of the present study may likely change with the future identification of new confounders. Second, it was a retrospective study conducted at a single facility; therefore, potential sampling bias may exist. The present study suggested that more frequent administration of APAP and higher doses per dose are necessary to maintain postoperative analgesia. Acetaminophen dosage of less than $4 \mathrm{~g} / \mathrm{d}$ was relatively safe, but there were reports of increased ALT at low dosage and racial differences in liver injury. ${ }^{26,27)}$ In this study, we also investigated the occurrence of drug-induced liver injury within one week after analgesic medication, but no cases occurred in both groups. DILI of APAP was reported to occur even after 1 week of administration, ${ }^{26)}$ therefore, the present 
results need to be validated by prospective safety studies.

\section{CONCLUSION}

In this observational study using a propensity score matching analysis, postoperative analgesic effects were slightly more potent in the LOX group than those in the APAP group. The use of PCA and the percentage of patients using postoperative rescue analgesics were also higher in the APAP than in the LOX group. In contrast, the increase in $\mathrm{SCr}$ after ORP was higher in the LOX group than in the APAP group. Therefore, it is important to focus more attention toward reduced renal function following ORP in patients receiving LOX than those receiving APAP.

APAP administration after ORP may be preferred to LOX administration, but the number of APAP doses and the dose per dose may need to be increased for patients with significant postoperative pain.

Conflict of Interest The authors declare no conflict of interest.

\section{REFERENCES}

1) Heidenreich A, Bellmunt J, Bolla M, Joniau S, Mason M, Matveev V, Mottet N, Schmid HP, Van Der Kwast T, Wiegel T, Zattoni F. EAU guidelines on prostate cancer. Part 1: screening, diagnosis, and treatment of clinically localised disease. Eur. Urol., 59, 61-71 (2011).

2) Harirforoosh S, Asghar W, Jamali F. Adverse effects of nonsteroidal antiinflammatory drugs: an update of gastrointestinal, cardiovascular and renal complications. J. Pharm. Pharm. Sci., 16, 821-847 (2013).

3) Ungprasert P, Kittanamongkolchai W, Price C, Ratanapo S, Leeaphorn N, Chongnarungsin D, Cheungpasitporn W. What is the "safest" non-steroidal anti-inflammatory drugs? Am. Med. J., 3, 115-123 (2012)

4) Blondell RD, Azadfard M, Wisniewski AM. Pharmacologic therapy for acute pain. Am. Fam. Physician, 87, 766-772 (2013).

5) Grams ME, Sang Y, Coresh J, Ballew S, Matsushita K, Molnar MZ, Szabo Z, Kalantar-Zadeh K, Kovesdy CP. Acute kidney injury after major surgery: a retrospective analysis of veterans health administration data. Am. J. Kidney Dis., 67, 872-880 (2016).

6) Romagnoli S, Ricci Z. Postoperative acute kidney injury. Minerva Anestesiol., 81, 684-696 (2015).

7) Chertow GM, Burdick E, Honour M, Bonventre JV, Bates DW. Acute kidney injury, mortality, length of stay, and costs in hospitalized patients. J. Am. Soc. Nephrol., 16, 3365-3370 (2005).

8) Ungprasert P, Cheungpasitporn W, Crowson CS, Matteson EL. Individual non-steroidal anti-inflammatory drugs and risk of acute kidney injury: A systematic review and meta-analysis of observational studies. Eur. J. Intern. Med., 26, 285-291 (2015).

9) Kanchanasurakit S, Arsu A, Siriplabpla W, Duangjai A, Saokaew S. Acetaminophen use and risk of renal impairment: A systematic review and meta-analysis. Kidney Res. Clin. Pract., 39, 81-92 (2020).

10) Breivik H, Borchgrevink PC, Allen SM, Rosseland LA, Romundstad L, Breivik Hals EK, Kvarstein G, Stubhaug A. Assessment of pain. Br. J. Anaesth., 101, 17-24 (2008).

11) Summary of recommendation statements. Kidney Int. Suppl.(2011), 2, 8-12 (2012).
12) Aithal GP, Watkins PB, Andrade RJ, Larrey D, Molokhia M, Takikawa H, Hunt CM, Wilke RA, Avigan M, Kaplowitz N, Bjornsson E, Daly AK. Case definition and phenotype standardization in druginduced liver injury. Clin. Pharmacol. Ther., 89, 806-815 (2011).

13) Austin PC. Balance diagnostics for comparing the distribution of baseline covariates between treatment groups in propensity-score matched samples. Stat. Med., 28, 3083-3107 (2009).

14) Shinoda S, Aoyama T, Aoyama Y, Tomioka S, Matsumoto Y, Ohe Y. Pharmacokinetics/pharmacodynamics of acetaminophen analgesia in Japanese patients with chronic pain. Biol. Pharm. Bull., 30, 157-161 (2007).

15) Onda A, Ogoshi A, Itoh M, Nakagawa T, Kimura M. Comparison of the effects of treatment with celecoxib, loxoprofen, and acetaminophen on postoperative acute pain after arthroscopic knee surgery: A randomized, parallel-group trial. J. Orthop. Sci., 21, 172-177 (2016).

16) Barr J, Fraser GL, Puntillo K, et al. Clinical practice guidelines for the management of pain, agitation, and delirium in adult patients in the intensive care unit. Crit. Care Med., 41, 263-306 (2013).

17) Kheterpal S, Tremper KK, Heung M, Rosenberg AL, Englesbe M, Shanks AM, Campbell DA Jr. Development and validation of an acute kidney injury risk index for patients undergoing general surgery: Results from a national data set. Anesthesiology, 110, 505-515 (2009).

18) Shin JI, Luo S, Alexander GC, Inker LA, Coresh J, Chang AR, Grams ME. Direct oral anticoagulants and risk of acute kidney injury in patients with atrial fibrillation. J. Am. Coll. Cardiol., 71, 251-252 (2018).

19) Mercado MG, Smith DK, Guard EL. Acute kidney injury: diagnosis and management. Am. Fam. Physician, 100, 687-694 (2019).

20) Agarwal PK, Sammon J, Bhandari A, Dabaja A, Diaz M, DusikFenton S, Satyanarayana R, Simone A, Trinh QD, Baize B, Menon M. Safety profile of robot-assisted radical prostatectomy: A standardized report of complications in 3317 patients. Eur. Urol., 59, 684-698 (2011).

21) Costalonga EC, Costa e Silva VT, Caires R, Hung J, Yu L, Burdmann EA. Prostatic surgery associated acute kidney injury. World $J$. Nephrol., 3, 198-209 (2014).

22) Krajewski ML, Raghunathan K, Paluszkiewicz SM, Schermer CR, Shaw AD. Meta-analysis of high- versus low-chloride content in perioperative and critical care fluid resuscitation. Br. J. Surg., 102, 24-36 (2015).

23) Bell S, Rennie T, Marwick CA, Davey P. Effects of peri-operative nonsteroidal anti-inflammatory drugs on post-operative kidney function for adults with normal kidney function. Cochrane Database Syst. Rev., 11, CD011274 (2018).

24) Lassnigg A, Schmidlin D, Mouhieddine M, Bachmann LM, Druml W, Bauer P, Hiesmayr M. Minimal changes of serum creatinine predict prognosis in patients after cardiothoracic surgery: a prospective cohort study. J. Am. Soc. Nephrol., 15, 1597-1605 (2004).

25) Hiragi S, Yamada H, Tsukamoto T, Yoshida K, Kondo N, Matsubara T, Yanagita M, Tamura H, Kuroda T. Acetaminophen administration and the risk of acute kidney injury: A self-controlled case series study. Clin. Epidemiol., 10, 265-276 (2018).

26) Watkins PB, Kaplowitz N, Slattery JT, Colonese CR, Colucci SV, Stewart PW, Harris SC. Aminotransferase elevations in healthy adults receiving 4 grams of acetaminophen daily: A randomized controlled trial. JAMA, 296, 87-93 (2006)

27) Heard K. Asymptomatic alanine aminotransferase elevations with therapeutic doses of acetaminophen. Clin. Toxicol. (Phila), 49, 90-93 (2011). 